Planetary Systems in the Universe - Observation, Formation and Evolution

Proceedings IAU Symposium No. 202, (C2004 IAU

Alan Penny, Pawel Artymowicz, Anne-Marie Lagrange, \& Sara Russell, eds.

\title{
Precision Astrometry with the Space Interferometry Mission
}

\author{
Stephen C. Unwin \\ Jet Propulsion Laboratory, California Institute of Technology, 4800 Oak \\ Grove Drive, Pasadena, CA 91109, USA
}

\begin{abstract}
SIM - the Space Interferometry Mission - will be the first of a new generation of space instruments using interferometry. Designed for precision astrometry, it will be a 10-meter optical interferometer providing 4 microarcsecond ( $\mu$ as) absolute position measurements over the whole sky, using a grid of stars (and quasars) for a reference frame. Maintaining its astrometric precision on stars as faint as 20 magnitude, it will far surpass the capability of ground-based astrometry, opening up a new era of space-based astrometry. A wide range of astrophysics problems will be addressed by SIM, including formation and dynamics of our Galaxy, calibration of the cosmic distance scale, and fundamental stellar astrophysics. SIM will search for planetary companions to nearby stars, by detecting the well-known astrometric 'wobble' signature with a single-measurement precision of $1 \mu$ as, enabling the detection of planets down to about an Earth mass. SIM will serve as a technology pathfinder for NASA's Terrestrial Planet Finder. Launch is currently planned for 2008, with a mission duration of 5 years.
\end{abstract}

\section{Science with SIM}

One of the most exciting of the mission goals for SIM is the detection of lowmass planets orbiting nearby stars. This rapidly-expanding field of astronomy is beginning to allow meaningful comparisons between our own Solar System, and those around other stars. SIM plays a crucial role, with the ability to detect planets as small as one Earth mass around the closest solar-type stars, with an astrometric accuracy of $1 \mu$ as after a 1-hour measurement. SIM will also perform the essential role of verifying the candidate target list for the Terrestrial Planet Finder (TPF) mission, which will search for possible tracers of biological activity on nearby planets. Unlike a survey mission, specific objects of interest can be scheduled for detailed observation, allowing studies of the kinematics and stability of multiple-planet systems. For details of the search space which SIM and other instruments will explore in the quest for understanding planetary systems, see the papers by the SIM team.

In its global astrometry (wide-angle) mode, SIM will address a variety of problems in stellar and Galactic astrophysics. SIM will provide $4 \mu$ as precision absolute positions for stars down to 20 magnitude and parallaxes to comparable accuracy. The proper motion accuracy will be about $2 \mu$ as /yr at the end of its 5year mission. The detailed science program will be defined by the Science Team appointed by NASA, but a summary of the science capability can be found in Peterson (2000). Among the possible topics are: calibration of the cosmic 


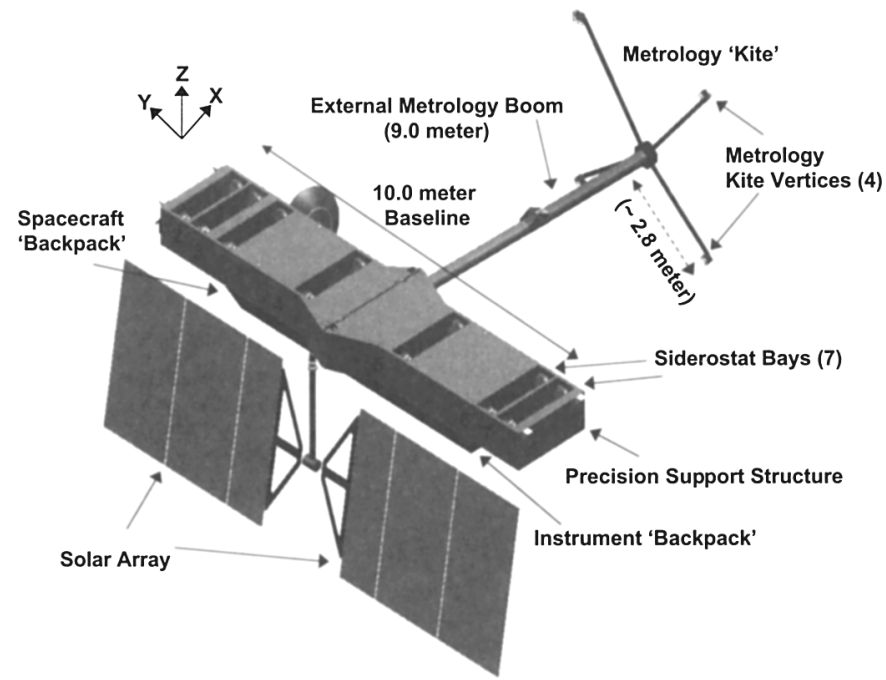

Figure 1. The SIM instrument, deployed into Earth-trailing solar orbit

distance scale using Cepheids and other indicators; ages of globular clusters; stellar dynamics of the Galaxy; orbits of globular clusters and satellite galaxies (including tidal streams); dynamics and evolution of binary stars; astrophysics of luminous stars; and rotational parallaxes of nearby galaxies.

\section{SIM Instrument and Mission}

The Space Interferometry Mission (SIM) will be a visible wavelength interferometer with a 10-m baseline (Danner \& Unwin 1999). It is designed to measure the position of white-light fringes to a small fraction of the basic fringe spacing. By avoiding the disturbing effects of the atmosphere, SIM can reach faint magnitudes even with modest collecting apertures. For its search for low-mass companions to nearby stars, SIM observes relative to nearby $\left(\leq 1^{\circ}\right)$ reference stars, with an accuracy of $1 \mu$ as after a 1-hour measurement. With repeated visits, SIM will detect the astrometric 'wobble' signature. For its global astrometry mode, SIM will develop and maintain a reference grid of $\sim 3000$ stars, with $4 \mu$ as positions, to which science targets will be referenced.

SIM is currently in its Formulation Phase, in which the basic design concept is being developed to the level where an accurate cost and schedule for construction and launch of the flight instrument can be made. Although significant details have changed in the design, the fundamental concept for SIM is three parallel simultaneous Michelson interferometers (Figure 1). Parameters defining the instrument and mission shown in Table 1. Two interferometers track fringes on bright guide stars, to stabilize the optical system, and a third observes science targets sequentially. Path lengths are matched using movable delay lines, to produce white-light fringes, and an internal laser metrology beam is used to measure the optical path difference which is the fundamental astro- 
metric quantity. An external laser metrology boom monitors the length and orientation of the three baseline vectors, and tracks changes during observation. Each siderostat has $15^{\circ}$ diameter field for target acquisition. Global astrometry is achieved by observing stars in common between overlapped fields.

Table 1. SIM Instrument and Mission Parameters

\begin{tabular}{ll}
\hline \hline Baseline & $10 \mathrm{~m}$ \\
Wavelength range & $0.4-0.9 \mu \mathrm{m}$ \\
Telescope aperture & $0.3 \mathrm{~m}$ diameter \\
Astrometric Field of Regard & $15^{\circ}$ diameter \\
Detector & $\mathrm{Si} \mathrm{CCD}$ \\
Orbit & Earth-Trailing solar orbit \\
Mission Duration & 5 years (launch 2008) \\
Astrometry (wide-angle) & $4 \mu \mathrm{as} \mathrm{mission} \mathrm{accuracy}$ \\
Limiting magnitude (V band) & $20 \mathrm{mag}$ \\
Astrometry (narrow-angle) & $1 \mu \mathrm{as}$ accuracy in 1 hour \\
\hline
\end{tabular}

In addition to precision astrometry, SIM is an important step on the technology path to large space-based interferometers, such as the Terrestrial Planet Finder (TPF Science Working Group 1999). SIM will demonstrate many important functions, such as the operation of a interferometer, which is intrinsically complex. It is planned to carry a nulling demo capable of on-axis starlight suppression to $10^{-4}$. SIM will demonstrate aperture synthesis imaging, using the proven method of radio astronomy imagers; it will be the first instrument to do this in space. Although only a demo using a small fraction of the mission time, SIM can provide high dynamic range images with a resolution of 10 milliarcsec.

The work described in this paper was performed at the Jet Propulsion Laboratory, California Institute of Technology, under a contract with the National Aeronautics and Space Administration. The SIM web site contains more information on this mission, technology development at JPL, and NASA's Origins scientific program ( http://sim.jpl.nasa.gov/ ).

\section{References}

TPF Science Working Group, 1999, Terrestrial Planet Finder (TPF), JPL Publication 99-3 ( http://tpf.jpl.nasa.gov/library/tpf_book/ )

Danner, R. M., \& Unwin, S. C. 1999, Space Interferometry Mission: Taking the Measure of the Universe, JPL Document 400-811 3/99 (Pasadena, California)

Peterson, D. M., \& Shao, M. 2000, SIM Science Working Group Final Report, JPL ( http://sim.jpl.nasa.gov/library/technical_papers.html ) 